\title{
Neuropsychology in Temporal Lobe Epilepsy: Influences from Cognitive Neuroscience and Functional Neuroimaging
}

\author{
Mary Pat McAndrews ${ }^{1,2}$ and Melanie Cohn ${ }^{1,2}$ \\ ${ }^{1}$ Krembil Neuroscience Centre, University Health Network 4F-409, 399 Bathurst Street, Toronto, ON, Canada M5T 2 S8 \\ ${ }^{2}$ Department of Psychology, University of Toronto, Toronto, ON, Canada M5S 1A1 \\ Correspondence should be addressed to Mary Pat McAndrews, mary.mcandrews@uhn.on.ca
}

Received 4 July 2011; Accepted 9 October 2011

Academic Editor: Seyed M. Mirsattari

Copyright ( 2012 M. P. McAndrews and M. Cohn. This is an open access article distributed under the Creative Commons Attribution License, which permits unrestricted use, distribution, and reproduction in any medium, provided the original work is properly cited.

\begin{abstract}
Neuropsychologists assist in diagnosis (i.e., localization of dysfunction) and in prediction (i.e., how cognition may change following surgery) in individuals being considered for temporal lobe surgery. The current practice includes behavioural testing as well as mapping function via stimulation, inactivation, and (more recently) functional imaging. These methods have been providing valuable information in surgical planning for 60 years. Here, we discuss current assessment strategies and highlight how they are evolving, particularly with respect to integrating recent advances in cognitive neuroscience.
\end{abstract}

\section{Introduction}

Neuropsychologists have been core members of epilepsy surgery teams since the 1950s. In his excellent historical review, Loring [1] notes that this began contemporaneously at centres at the University of Illinois, the University of London, and the Montreal Neurological Institute/McGill University; at each institution, there was a close collaboration amongst surgeons, neurologists, and neuropsychologists. As these surgeries were performed in many instances "blind" to any structural abnormality underlying the seizure focus, the initial task of the neuropsychologist was to provide converging evidence for localization, together with EEG (and later CT/MRI), by charactering the focal functional deficit. Another key objective was to use the assessment to predict how function might change following surgery in an attempt to avoid catastrophic cognitive losses; this was a task for which neuropsychology was uniquely qualified. Prediction of more subtle changes also required a substantial investment in systematic postsurgical assessments that would provide the evidentiary basis for those predictions, and this is an important legacy of Brenda Milner at the MNI, whose contributions to the field are widely appreciated.

The goals of localization/diagnosis and prediction of change remain at the forefront of neuropsychology practice in epilepsy surgery programs, which relies primarily on the use of standardized neuropsychological tests with proven sensitivity and specificity in this population. However, there is a growing appreciation that additional information from tools such as structural MRI (e.g., presence/absence of mesial temporal sclerosis) need to be incorporated into our models to improve accuracy. Furthermore, neuropsychologists recognize that characterizing both the functional integrity of the tissue to-be resected as well as the functional reserve of the rest of the brain to a high level of accuracy for the individual patient requires that we continue to develop more sophisticated conceptualizations and analytic tools. In particular, as we come to understand how cognitive operations such as memory are supported by somewhat flexible and overlapping brain networks, new modes of analysis are required. Finally, recent data clearly show both that there is a fair degree of reorganization of these networks in patients with temporal lobe epilepsy (TLE) and that even when seizures are exclusively in mesial temporal regions, there is more widespread anatomic and functional disturbance [2]. In our view, this means that the neuropsychology of epilepsy is at, perhaps, another "landmark" period in its development. In this paper, we share some of our thoughts regarding the state of the art and the ways forward in that trajectory. 


\section{Neuropsychological Assessment of Memory}

In general terms, the neuropsychological evaluation of individuals with epilepsy involves the administration of an extensive battery of standardized behavioural tests tapping multiple cognitive domains (intellectual abilities, attention, visuospatial skills, language, executive function, and memory) with an emphasis on laterality [3]. In temporal lobe epilepsy, while determining language lateralization is a priority, extensive memory testing has been at the core of this assessment, initially motivated by the report of severe postoperative memory decline in two individuals who underwent unilateral resection, $\mathrm{PB}$ and FC [4]. Their devastating memory outcome was attributed to dysfunction of their remaining right temporal lobes given that their memory decline was of similar magnitude to that noted in HM who underwent bilateral temporal resections [5]. These findings highlighted the importance to test for functional reserve of the contralateral mesial temporal lobe, in addition to test the functional adequacy of the to-be-resected temporal lobe. Some of the typical standardized tests of memory and language used in presurgical assessments are described in Table 1; more complete descriptions, specific test citations, commentaries, and norms can be found in a comprehensive compendium of neuropsychological tests [6].

\section{Material Specificity}

A key concept in assessing the function of each temporal lobe "independently" is the material-specificity principle which proposes that verbal memory is a function of the left temporal lobe and visual or spatial memory is a function of the right temporal lobe [7]. In practice, the neuropsychologist compares an individual's verbal memory and visual memory to normative data as well as to each other. The presence of an asymmetry provides information on lateralization of dysfunction (e.g., left for poor verbal), and performance on the nonepileptic temporal lobe provides information on reserve. Impaired performance on the latter would raise concerns of significant risk to memory postoperatively and additional investigations (e.g., the intracarotid amobarbital procedure or IAP) would be indicated. The level of functional integrity of the to-be-resected temporal lobe also helps predict postoperative outcome as patients with intact preoperative function have "more to lose" following surgery relative to those who show a memory asymmetry concordant with EEG and/or MRI findings.

While the material specificity principle generally holds true, especially with respect to verbal memory $[8,9]$, its reliability in identifying laterality of dysfunction is not perfect. Saling [10] identified two main issues. First, sensitivity of verbal memory tests to left mesial temporal damage is variable across instruments, suggesting that verbal memory is not a unitary construct. For instance, people with left TLE (but not right TLE) show impaired memory for word pairs but have intact memory for prose passages [11]. Second, many visual memory tests lack both specificity and sensitivity to right TLE. Some studies do show a relationship between right TLE and visual memory [12-14], but others do not or show impairments of similar magnitude in both left and right TLE [15].

Additional concepts from contemporary neuroscience may supplement the material specificity principle and improve our ability to characterize functional adequacy and reserve. Some of these can be applied currently in test selection and interpretation (at least with respect to localization), as well as in the development of new standardized clinical tests, although much of this translational work remains to be done. Several models propose that the temporal lobes are functionally heterogeneous; different memory systems, processes, or types of information rely on different regions of the temporal lobes. Here, we review distinctions between episodic and semantic memory, relational and nonrelational processes, as well as the hippocampus' involvement in pattern separation and consolidation.

\section{Semantic versus Episodic Memory}

Declarative memory includes two memory systems, namely, semantic memory (i.e., conceptual knowledge about words, the world and the self) and episodic memory (i.e., memory for personally experienced events with a spatiotemporal context [16]). Based, in part, on double dissociations noted in patients with mesial temporal lobe (MTL) damage or disease and with frontotemporal dementia (e.g., [17]), it is widely accepted that the lateral temporal neocortex is critical for semantic memory and MTL regions, such as the hippocampus, support episodic memory. This division is reflected in current neuropsychological practice as typical evaluations include tasks of episodic memory (e.g., list learning) and of semantic knowledge (e.g., naming, verbal fluen$\mathrm{cy}$ ), which are interpreted as reflecting function of mesial and lateral temporal regions, respectively.

However, the two memory systems have a certain degree of interdependence or collaboration that varies as a function of task characteristics and demands (for review see [18]). This overlap provides a parsimonious explanation for the variable sensitivity of verbal "episodic" memory tests to left TLE because this sensitivity is proportional to the degree to which the material is semantically rich or consists of novel relationships. For instance, test sensitivity to left TLE is greater for list learning tests containing unrelated words or arbitrary word pairs (e.g., RAVLT or hard pairs on Paired Associates subtest of the WMS) than for semantically related ones (CVLT or easy pairs on the WMS), [19, 20]. Similarly, memory for prose passages, which is highly semanticized, relates to the integrity of the lateral neocortex rather than that of the hippocampus [11] (see Table 1 for further description of tests).

In these examples, semantic memory clearly contributes to performance on episodic memory tasks, but the reverse influence is also true. In a group of MTL amnesic individuals, Greenberg et al. [21] demonstrated deficits in semantic fluency that were of greater magnitude for categories that generally elicited autobiographical retrieval strategies in healthy controls (e.g., imaging their own kitchen when asked to generate a list of kitchen utensils rapidly) relative to those that did not. Of interest, functional neuroimaging of this 
TABLE 1: Neuropsychological tests in common use in epilepsy surgical centres.

\begin{tabular}{|c|c|}
\hline Test name/domains & Description \\
\hline \multicolumn{2}{|l|}{ Verbal memory } \\
\hline $\begin{array}{l}\text { California Verbal } \\
\text { Learning Test-II } \\
\text { (CVLT-II) }\end{array}$ & $\begin{array}{l}\text { Examinees recall a list of } 16 \text { words from } 4 \text { categories (furniture, vegetables, ways of travelling, and animals) after } \\
\text { each of } 5 \text { learning trials. Retention is assessed by free recall and cued recall of the list following the presentation } \\
\text { of interfering material and following a } 20 \text {-minute delay period, as well as by delayed yes-no recognition. }\end{array}$ \\
\hline $\begin{array}{l}\text { Rey Auditory Verbal } \\
\text { Learning Test (RAVLT) }\end{array}$ & $\begin{array}{l}\text { Examinees recall a list of } 15 \text { unrelated words after each of } 5 \text { presentations. Retention is assessed by free recall } \\
\text { of the original list after a second list is presented and following a } 20 \text {-minute delay period, as well as by delayed } \\
\text { yes-no recognition trial or a recognition trial involving recognizing the studied words embedded in a prose. }\end{array}$ \\
\hline $\begin{array}{l}\text { Verbal Paired Associates } \\
\text { I \& II } \\
\text { subtests-Wechsler } \\
\text { Memory Scale (WMS), } \\
\text { WMS-R, WMS-III, and } \\
\text { WMS-IV }\end{array}$ & $\begin{array}{l}\text { Measures relational memory of word pairs over repeated learning trials. After each presentation of the list } \\
\text { of pairs, the first word is given and the examinee is required to provide its associate. Errors are corrected } \\
\text { immediately. The format of the test has changed across versions in terms of number of pairs (from } 8 \text { to } 14 \text { ), } \\
\text { types of pairs (both easy/related pairs and hard/unrelated pairs in most versions), number of learning trials ( } 3 \\
\text { to 6), and the inclusion of a 20- to 30-minute delayed cued recall and delayed recognition trials (present since } \\
\text { WMS-R). }\end{array}$ \\
\hline
\end{tabular}

Logical Memory I \& II subtests-WMS, WMS-R, WMS-III and WMS-IV

Names subtest-Doors \& People Test

Words

subtest-Recognition

Memory Test
Consists of immediate recall and delayed recall of two orally presented prose passages as well as yes-no delayed recognition of story elements. Some changes in test format and content have been introduced across versions.

Examinees read two lists of names (12 per list) and recognize these names on a four-alternative forced-choice recognition task.

Examinees rate 50 words as pleasant or unpleasant and recognize these words on a two-alternative forced-choice recognition task.

Visual memory

Rey-Osterrieth Complex This task involves copying a complex figure, which provides a measure of construction skills and planning, Figure followed by an unexpected recall and recognition tests given at various delays depending on the center's protocol.

Visual Reproduction I \&

II subtests-WMS,

Four or five visual designs are shown for 10 seconds each and are reproduced from memory. Delayed WMS-R, WMS-III, and reproduction and yes-no recognition are also done 20- to 25-minutes after learning.

WMS-IV

Designs I \& II

(WMS-IV)

Faces I \& II

subtests-WMS-III

Faces

subtest-Recognition

Memory Test

Doors subtest-Doors \& People Test

Language

Boston Naming Test

Assesses visual confrontation naming using line drawings of common objects. Semantic and phonemic cues are provided for items that are not named within 20 seconds. Versions of different lengths exist (15, 30, and 60 items).

Assesses the spontaneous production of words under particular search rules over a set period of time (usually 60 Verbal Fluency particular letters (e.g., F, A, and S) and belonging to particular categories (e.g., animals, fruits, and vegetables), respectively. Other rules have also been used, including proper names and actions.

task with healthy controls reveals bilateral mesial temporal activation, suggesting that both dominant and nondominant MTL regions are engaged [22]. In TLE, semantic fluency deficits are typically interpreted as dysfunction of the left lateral neocortex, but the above findings raise the possibility that hippocampal dysfunction also may contribute to certain impairments in semantic access, including the nondominant MTL. This certainly provides a compelling explanation of findings showing impaired semantic fluency not only in left TLE, but also in right TLE individuals with hippocampal (but not neocortical) involvement [23]. Another example wherein episodic memory enhances semantic retrieval 
pertains to memory for famous people. Fame judgment and speeded reading tasks of famous names were shown to be facilitated for those associated with autobiographical significance (e.g., most people recollect a personal event related to John F. Kennedy) in healthy controls and individuals with semantic dementia, but not in MTL amnesic individuals [24]. Although autobiographical significance of the material used was not investigated directly, it may have contributed to the deficit noted on a famous people memory task, which was of equal severity in left, right, pre- and postoperative TLE individuals [25].

In sum, the separation between episodic and semantic memory is less distinct than previously thought and the degree to which semantic memory contributes to a given episodic task, and vice versa, need to be considered in test selection and interpretation. Selecting tasks with various degree of respective contribution from semantic and episodic memory may help characterize the source of potential difficulties.

\section{Relational versus Nonrelational Memory}

Within episodic memory, several dissociations have been found between memory of nonrelational information (e.g., single words or objects) and memory for relational information (e.g., pairs of single elements such as word-word or word-object). According to dual process models, the hippocampus is crucial for relational memory and recollection which enable retrieval of contextually rich events, while adjacent mesial temporal regions (e.g., perirhinal cortex) can support nonrelational memory via familiarity, which is a process characterized by a decontextualized feeling of oldness or of prior exposure (for review see $[26,27]$ ). These memory processes typically work in concert but are preferentially called upon in tasks involving particular types of material or test format. Tasks of free recall, novel pair associates, source memory, and autobiographical memory are more recollection based, while tasks of single item recognition can be supported by familiarity. Experimental procedures have also been devised to extract purer indices of each process [27].

Some studies tested relational memory/recollection in TLE using a variety of material (sounds, faces, spatial locations, words, and descriptions of personally experienced autobiographical events) and test formats, but only a few of these also assessed familiarity. As expected given hippocampal involvement, all studies showed relational memory/recollection deficits in TLE and, in most of these, the deficits were present regardless of seizure or excision laterality [28-33], although these deficits were material specific in others $[20,34]$. With respect to familiarity, it was intact in one study [34] and impaired for material processed by the damaged hemisphere in others $[28,30]$. This deficit is likely related to perirhinal lesions or dysfunction based on findings with $\mathrm{NB}$, who, following a temporal lobe resection that spared the hippocampus, but not the surrounding regions (amygdala, entorhinal and perirhinal cortex, and lateral temporal neocortex), demonstrated impaired familiarity but intact recollection [35].
Together, these findings suggest that relational memory tasks may provide information on the functional integrity of the hippocampus, although they may not consistently provide information on laterality. Tasks that rely to a greater extent on familiarity may be more lateralized, consistent with the material specificity principle, and be indicative of dysfunction of the perirhinal cortex. These principles can be applied to selection of current tools, for instance, when contrasting performance on free recall tasks (which are more recollection based) and forced-choice recognition (which are more familiarity based).

\section{Pattern Separation}

Computational models of memory, such as the complementary-learning-systems model [36, 37], proposed that the neocortex contributes to memory by slowly extracting general information over repeated similar experiences while the hippocampus allows quick encoding of specific episodes as distinct representations. The latter is achieved via a pattern separation operation which minimizes the overlap between similar representations. Subregions of the hippocampus, including the dentate gyrus and its projection to CA3, are specifically involved in pattern separation. CA1 and extrahippocampal regions, such as the entorhinal cortex, support retrieval via pattern completion, which allows reconstruction of a memory representation when provided with a fragment of the original studied item or events [38]. Pattern separation enables one to discriminate between highly similar items or experienced events at retrieval and to distinguish them from similar lures. While this model is most studied in animal models, a case study with an individual with damage restricted to the hippocampus [39] and a high-resolution fMRI study in healthy participants confirmed this functional division using a recognition task in which lures are highly similar to targets [40].

To our knowledge, pattern separation has not been systematically investigated in TLE, but some results in two studies with postoperative TLE may be interpreted as evidence of significant deficits with this operation. In one study, we used an associative recognition task in which participants had to discriminate studied word pairs from lures composed of studied words rearranged in a novel way [28]. Interestingly, TLE patients had significantly greater difficulties rejecting these overlapping lures than endorsing studied pairs, which requires a recall-to-reject strategy (i.e., retrieving the original pairings in order to reject the current rearranged one) thought to depend on pattern separation. In another study, [41] left and right postoperative TLE were impaired on verbal and visual standardized recognition tasks, respectively (Doors \& People Test), in which lures and distractors are very similar to one another. Interestingly, the effect size for the visual subtest was markedly greater than that of the verbal subtest (cohen's d of 2.05 for doors versus 0.54 for names) and those typically reported with visual memory tasks [42].

Further studies, especially with preoperative patients, may ultimately inform us about dysfunction in specific 
subregions of the hippocampus. Until then, findings on pattern separation can inform test selection and interpretation as we expect that, due to hippocampal dysfunction, TLE will be associated with greater difficulties on tasks including highly overlapping and interfering material than tasks in which material is distinct.

\section{Consolidation}

The hippocampus is critical for consolidation in declarative memory, which refers to the stabilization of long-term memory and enables retention of information over time. There are two types of consolidation: (1) synaptic (or cellular) consolidation, which is completed within minutes to hours, applies to all memory systems and species and consists of long-term modification of synaptic proteins, synaptic remodeling, and growth, and (2) system consolidation, which takes place over days or years and pertains to memories that are dependant on the hippocampus initially, but become independent from this structure and "transfer" to the neocortex after undergoing reorganization (for review see [43]). It is well known that individuals suffering from amnesia, such as HM, have impaired retention, even at short delay periods [5]. TLE patients (especially left) often show milder, but significant forgetting after a few minutes or hours [44], which suggests that synaptic consolidation is reduced. Recent studies have also reported accelerated longterm episodic forgetting in TLE (but not semantic), that is, significant forgetting of information following days or weeks despite intact initial learning and retention at shorter delays (30-60 minutes) $[45,46]$.

Thus, system consolidation and synaptic consolidation within the episodic memory system appeared to be dissociated in TLE. Importantly, this illustrates that current standard neuropsychological assessments may not capture potential hippocampal dysfunction given that no standardized task include delays that are much longer than 30 minutes.

\section{Prediction: Combination of Investigations}

Thus far, we have discussed concepts that have the potential to improve our ability to assess both functional reserve and adequacy of the temporal lobes behaviourally and, ultimately, improve prediction of postoperative outcome (although no studies have assessed this directly in a single cohort). While preoperative behavior (e.g., memory function) is informative, higher predictive validity is obtained when it is combined with certain types of clinical data (e.g., age of onset, presence of mesial temporal lobe sclerosis (MTS), or cortical dysgenesis on MRI) [47].

The intracarotid amobarbital test (IAP) has been used to predict the consequences to memory of planned temporallobe resections since the 1960s $[48,49]$. This procedure, in which an anesthesizing agent is injected into the cerebral vasculature of one hemisphere, is intended to evaluate risk by evaluating memory encoding abilities of the contralateral hemisphere (i.e., functional reserve) and ipsilateral hemisphere (i.e., functional adequacy) independently. In cases of failure on contralateral testing or "crossed asymmetry" (i.e., better performance from the ipsilateral than the contralateral test), surgical planning was modified or in some cases surgery foregone in order to minimize cognitive morbidity. However, in recent years, the added value of the IAP has been brought into question, as studies have indicated that there is a relatively high base rate of failure or for crossed asymmetry, particularly associated with dysphasia following dominant-hemisphere injection, without significant postoperative complications $[50,51]$. Furthermore, in larger series of "uncomplicated" TLE (i.e., those in whom there was no evidence of significant bitemporal dysfunction), studies employing multivariable regression techniques have found that IAP results have little or no added predictive value when preoperative memory scores and structural MRI findings are considered $[52,53]$. Thus, given the questionable utility of IAP in many circumstances and given the invasiveness of the procedure, there is growing consensus that its use must be carefully considered on a case-by-case basis and perhaps used only in circumstances in which the risk of postoperative amnesia is considered high on the basis of all other available evidences [54]. At the same time, there is a rapidly evolving literature on functional MRI (fMRI) for assessing both language and memory capacities, providing a noninvasive alternative to the IAP, to which we now turn.

\section{Functional Neuroimaging}

Although there is an older literature including positron emission tomography and single-photon emission computed tomography imaging in TLE, the majority of studies in which imaging has been used to evaluate cognitive function use fMRI. Functional brain mapping with MRI, in which the blood-oxygen-level-dependent (BOLD) signal provides an indirect measure of the distribution of neuronal elements activated in a task, has been used in cognitive neuroscience since the early 1990 s $[55,56]$. Its use clinically is a newer phenomenon, starting with attempts to map language and sensorimotor functions in patients with tumours and epilepsy where other methods of evaluating regional involvement in these processes (IAP, stimulation mapping) could be used to validate the fMRI results. It has proven so successful for sensorimotor and language mapping that there are now (since January 2007) billing codes that permit compensation for such procedures in the US healthcare system. Its effectiveness in determining the functional adequacy of mesial temporal structures for supporting memory, the next logical application in epilepsy surgery, is not yet validated as the reliability of prediction vis-à-vis surgical outcome has yet to be established. Here, we discuss some of the relevant research and highlight some of the challenges to functional mapping posed by network dynamics and functional reorganization in epilepsy. We also alert the reader to several excellent recent reviews of some of this material $[57,58]$.

\section{0. fMRI for Language}

Functional MRI is now used in many centres for lateralization and localization of language functions so as to avoid 
significant postoperative aphasias. Whilst there is no expectation that there will be a huge variation in the "canonical" language regions in the TLE population, the literature does suggest a higher incidence of atypical dominance (or greater engagement of the typically "nondominant" hemisphere) in epilepsy patients. This is particularly true in cases of early brain injury resulting in weaker right-hand dominance, as was previously established by inactivation procedures such as stimulation mapping and the IAP $[59,60]$. Of interest, the proximity of a lesion to the primary receptive and expressive regions (Wernicke's and Broca's, resp.) in the left hemisphere does not appear to be a predictor of such reorganization [61, $62]$, suggesting that atypical dominance may reflect a more subtle disruption of language networks. Thus, it is important to map these language networks in individuals even when the planned resection would be considered unlikely to impact the primary regions known to be involved in language processing.

A number of different tasks have been used for language mapping in fMRI, including ones that emphasize expressive functions such as naming and fluency as well as those tapping receptive abilities such as word/nonword decisions or sentence comprehension. Expressive tasks are typically done without overt speech due to concerns about head motion. There is no "standard" paradigm, and while various papers argue the effectiveness of one over another (e.g., $[63,64])$, there is a growing acceptance of the use of a panel of tasks that tap both expressive and receptive functions, as combining these improves greater sensitivity and specificity $[65,66]$. For lateralization, the most commonly used metric is an asymmetry index (Left - Right/Left + Right) which is typically calculated on the number of voxels that exceed a particular activation threshold. The calculation is performed either on entire hemisphere or on regions of interest in frontal and temporal lobes typically associated with language processing. Although cutoffs vary for classification of dominance, ratios of +0.25 and -0.25 are frequently used to describe left and right dominance, respectively, with intermediate values indicating weaker lateralization. Figure 1 shows activation maps for two TLE patients in whom our fMRI task panel (verb generation, category fluency, sentence completion, and naming to description) demonstrated left or right hemisphere language dominance, illustrating its utility at the individual subject level.

Overall, there is quite good agreement between fMRI using asymmetry indices and IAP results for language dominance $[65,67-69]$. In the largest series to date comprised of 100 epilepsy patients, Woermann et al. [70] found over 90\% concordance between fMRI and IAP estimates of language lateralization, although classification agreement was somewhat poorer for extratemporal epilepsy in the dominant hemisphere $(25 \%$ discordance, fMRI suggesting bilateral representation). As with other methods (IAP, dichotic listening), fMRI reveals more atypical language lateralization in individuals with weaker right-hand dominance [71]. Nonetheless, in right-handed patients with left TLE, up to $30 \%$ of individuals show bilateral or right hemisphere language organization by fMRI, a rate that is higher than in healthy controls or patients with right TLE [72-74]. This shift appears to be more prominent in the temporal than frontal lobes, and it is correlated with age of onset of damage or intractable epilepsy in that region [75], suggesting a direct impact of seizures on lateralization. Of note, this may also be a reflection of damage to pathways connecting temporal and frontal brain regions in epilepsy as shown by recent evidence from diffusion tensor imaging (DTI) [76].

Determining whether a particular region is critical for language (e.g., a region in close proximity to a structural lesion or an epileptic focus) presents a bit greater difficulty than determination of dominance, as here one must have a means of validating that the task-related activation signifies performance capacity rather than mere correlation or association. Correspondence with stimulation mapping provides one avenue for validation that can avoid the consequences of a postoperative aphasia. Rutten and colleagues [77] reported that a combination of tasks (verb generation, sentence comprehension, and picture naming) was highly sensitive for detection of critical sites, based on disruption of speech with direct stimulation. However, as there were "false positives" (regions in which activation was apparent with no speech disruption) in many cases, the authors felt that fMRI could supplement rather than replace stimulation, perhaps to guide stimulation targets. A recent review of the utility of the IAP in contrast to fMRI reaches a similar conclusion [78]. Nonetheless, activation in the dominant temporal lobe has been shown to be correlated with postoperative naming decline following left anterior temporal lobectomy [79] and there are some impressive instances in which fMRI shows interhemispheric dissociation between frontal and temporal lobe language regions which are not identified by IAP [ 80 , 81].

In addition to alterations in hemispheric dominance in patients with TLE, there is evidence of intrahemispheric reorganization of language. Several studies have reported increased prefrontal activation, which may reflect compensatory processes given that it is seen when performance is unimpaired $[82,83]$. Such findings suggest alterations in the components and operation of language networks in TLE, and recent studies have examined directly the connectivity of nodes within these networks. Waites and colleagues reported reduced resting-state connectivity amongst frontal and temporal nodes in patients with left TLE [84]. Protzner and McAndrews [85] used partial least square (PLS) analysis, a multivariate technique, to examine networks that support performance on a test of confrontation naming. Critically, the networks were defined by activation during a verb generation task and the criterial naming task performance was measured weeks to months before or after scanning. They found three separate patterns relating brain activation to naming performance, one each for controls, patients with left TLE, and patients with right TLE. Thus, there may be fairly complex patterns of reorganization associated with TLE, and exploration of these may be important in predicting the nature and degree of postoperative changes following epilepsy surgery. 

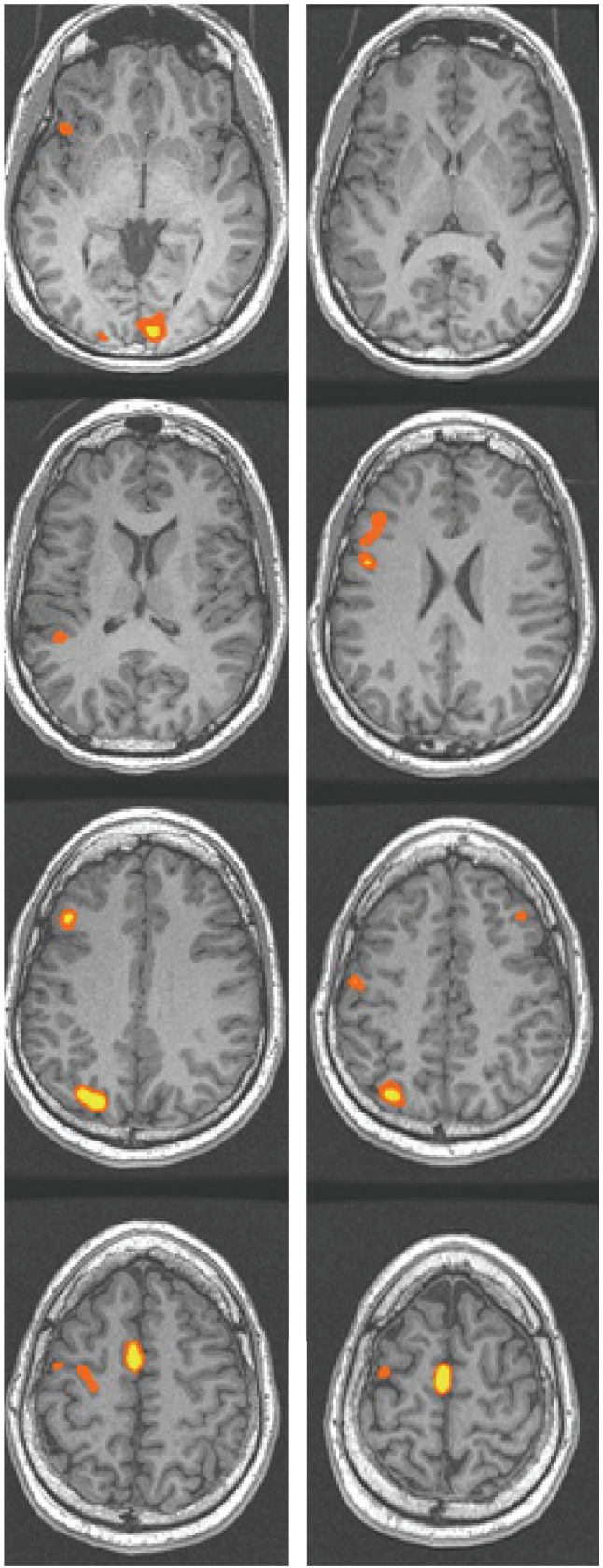

(a)
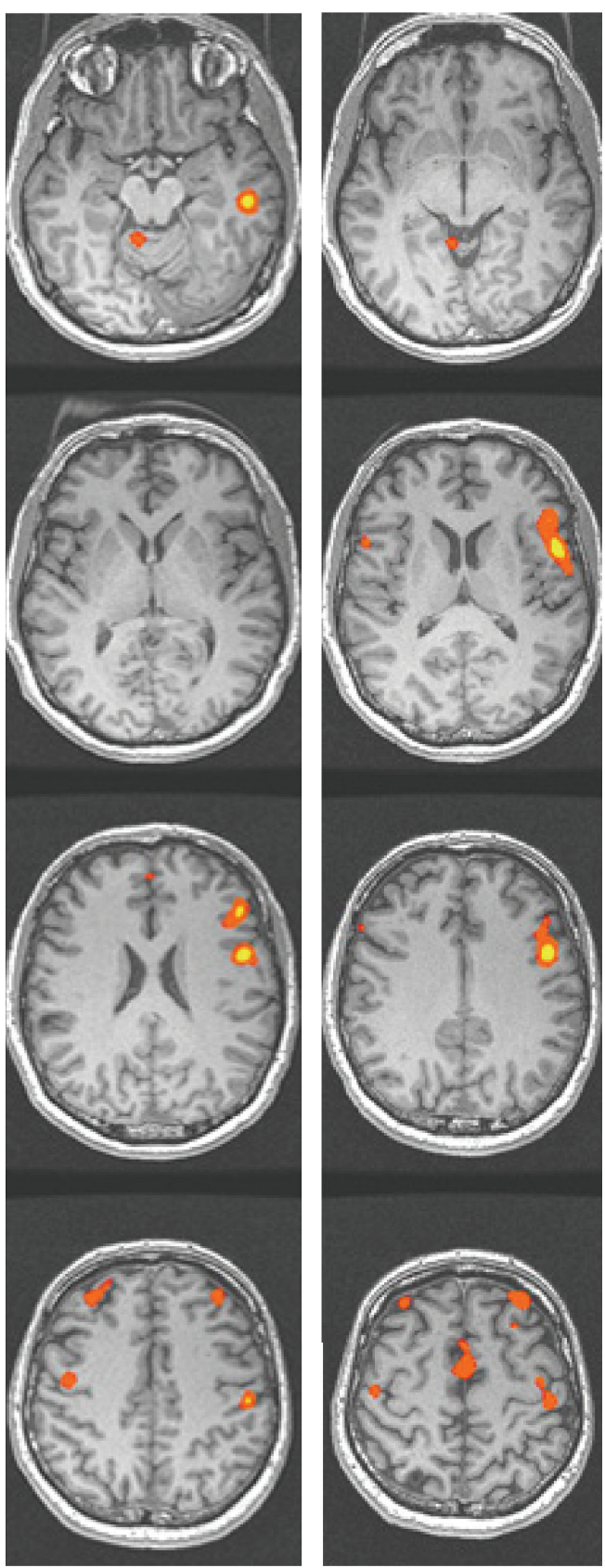

(b)

FIgure 1: Presurgical fMRI for language in patients with TLE. Two individuals with epileptic foci in the left temporal lobe were scanned using a panel of tasks involving 25-second blocks of verb generation, sentence completion, category fluency, and naming to confrontation, alternating with 20-second blocks of fixation. The case in (a) shows left-hemisphere dominance, and the case in (b) shows right hemisphere dominance. In figure, L: left and R: right; threshold is set to $z>2.25$.

\section{1. fMRI for Memory}

Although the past 20 years of fMRI research in cognitive neuroscience have yielded important insights into brain regions and networks supporting memory processes, its application in a clinical context, particularly in ascertaining the functional integrity of mesial temporal structures in TLE, is limited. There are a number of reasons for this limitation, including the complexity of memory operations and their relative dependence on specific brain structures: the same area may be involved in support encoding and retrieval but less so for recognition. Like behavioral tasks reviewed above, activation tasks should reliably discriminate between dominant and nondominant temporal lobes and between mesial and lateral temporal (in addition to frontal and parietal lobe) contributions to memory performance. 
Yet, even material specificity is less reliably seen with memory activation paradigms than one would expect from the lesion data [86]. Finally, it is important to establish a relationship between functional adequacy of the region to-be-resected and some characteristics of the BOLD signal (i.e., do 3 activated voxels represent a small risk of postoperative decline whereas 20 voxels signify a large risk?). The field is not sufficiently mature to have highly reliable activation tasks with appropriate cutoffs for clinical decision making. However, there have been some important strides in this regard, as we review here.

In attempting to assess functional integrity in the temporal region, one strategy has been to use tasks in which there is typically bilateral MTL activation in controls and assess whether there is reduced activation ipsilateral to the epileptic focus and thus greater asymmetry in TLE patients. A number of studies fulfill those criteria, typically using encoding or retrieval of complex visual material such as scenes, routes, autobiographical memories, and demonstrating reduced ipsilateral activation [87-90]. Of note, there are issues as to their sensitivity to hippocampus versus cortical MTL regions [91]. Another strategy is to use material-specific memory probes that are more likely to preferentially activate one or the other mesial temporal region (e.g., words for the left hemisphere, abstract designs for the right hemisphere); these may also exploit associative or relational memory tasks that have been shown to be particularly good at activating the MTL. For example, Dupont and colleagues using a verbal encoding and retrieval task found reduced MTL activation in patients with left MTS [92]. Using both verbal and visual material at encoding, some studies have consistently reported increased MTL activation in the side contralateral to the seizure focus $[93,94]$, although as noted above the material specificity can be difficult to demonstrate reliably in healthy controls [86]. Overall, these findings suggest that memoryinduced activation may be a sensitive marker of epileptic disturbance.

A crucial concern for fMRI is identifying specific parameters of activation that can characterize the functional integrity of the mesial temporal region, that is, parameters which correlate with other measures of function (e.g., neuropsychological tests, IAP results) and predict memory change following surgery. In that regard, there are some positive results in the literature but perhaps less than one might expect in contrast to the fairly robust language fMRI literature. This may be partly a function of the difficulties posed by signal loss and distortion in the basal temporal region and, importantly, by loss of fidelity caused by postprocessing choices in both metrics and standardization of patient data to normative structural templates [95]. While we must be mindful that it is impossible to know how many null findings there are on this question as they tend to be unpublished and left in "file drawers," the positive findings stand at least as proof of concept for the clinical utility of fMRI for memory assessment in TLE patients.

Several studies have reported good correspondence between asymmetry in MTL activation and IAP asymmetries $[87,93]$. Others have found clinical memory test performance to correlate positively with the magnitude of

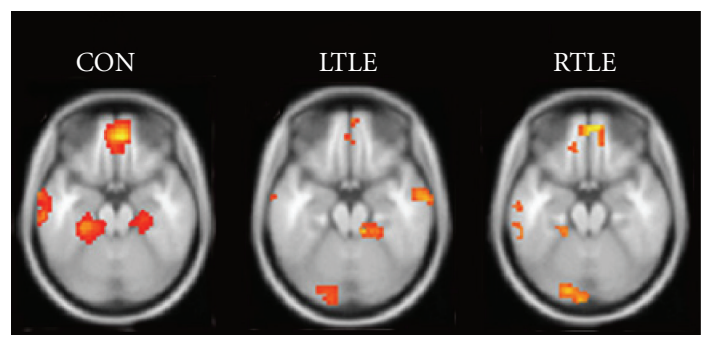

FIgURE 2: Autobiographical memory profiles. fMRI maps illustrate regions demonstrating higher activation for during autobiographical memory recall (retrieving personal memories based on event cues) relative to semantic retrieval (sentence completion). Note that there is less activity in the epileptogenic mesial temporal region for both left and right TLE groups compared to healthy controls $(n=10$ per group). In figure, L: left and R: right; threshold set at $P<.0001$, uncorrected.

activation in the MTL ipsilateral to the seizure focus [90, 96]. Rabin and colleagues [97] were the first to show that mesial temporal activation (during scene encoding) correlated with postoperative memory performance (on the scene recognition task); increased activation ipsilateral to the seizure focus was associated with greater decline. The crucial validation is, of course, whether activation can be used to predict clinically relevant postoperative memory compromise. There have been several studies demonstrating correlations between activation magnitude in the to-beresected MTL and the amount of memory change, with greater preoperative activity (expressed either as an absolute magnitude or asymmetry relative to the other MTL) associated with a more dramatic decline in material-specific memory function [96, 98-101]. However, there are also negative instances in which hippocampal activation may be reliable for a given task but does not predict postoperative memory change [102]. Furthermore, the extent to which fMRI parameters provide independent or additional power to the prediction of risk to memory, beyond factors such as neuropsychological test data and measures of hippocampal atrophy, has not been adequately addressed in a sufficient number of studies (see [103, 104]).

Our own work with autobiographical recall in patients with TLE illustrates the complexity of the relationship between functional activation during memory paradigms and clinical indicators. Retrieval of episodes from the personal past is well established as a powerful activator of mesial temporal regions $[105,106]$, and patients with TLE show marked deficits in recall of details of autobiographical experiences [29, 31]. Furthermore, mesial temporal damage in these patients results in a marked reduction in the affected region as well as the whole autobiographical network [89]. Figure 2 displays newer (unpublished) findings from our clinic in which a second cohort of left and right TLE patients showed reduced activation in the epileptogenic hippocampus as well as other regions in the network. Additionally, Maguire and colleagues showed similar alterations in hippocampal engagement in two other patients with mesial temporal damage from hypoxia $[107,108]$. Nonetheless, we have 
found relatively poor correspondence between the magnitude or extent of hippocampal activation and performance on clinical memory tests and even some negative correlations in certain tasks (unpublished observations). Of course, it is entirely possible that there is a nonmonotonic function relating activation to clinical impairment in TLE. Indeed, at least one study has reported increases in MTL activation in TLE patients associated with dysfunctional tissue [109]. Sperling and colleagues have observed this in other cases of mesial temporal damage in that patients with very mild cognitive impairment show hyperactivation in the MTL whereas patients with more severe memory deficits show hypoactivation compared to controls $[110,111]$.

More recent analyses of functional activation patterns have begun to focus on connectivity and network characteristics, rather than focal activation as an important determinant of the effects of TLE on memory. Our study of autobiographical recall demonstrated a significant change in connectivity throughout the autobiographical network, in that patients with left TLE showed reduced connectivity with mesial temporal regions and enhanced connectivity between anterior and posterior midline regions [89]. Of interest, several studies involving patients with left TLE have shown strong correlations between language asymmetry and memory change $[102,112]$, suggesting that a more widespread pattern of dysfunction is reflected in memory deficits found in epilepsy. Indeed, it is likely that a full appreciation of functional competency, and thus the likelihood of functional impairment following surgery, requires consideration of brain networks supporting memory and language rather than focusing exclusively on MTL activation. As noted above, we found that TLE patients and controls activated the same networks in a language task but that there were subtle differences in the network components associated with performance on a clinical test [85]. Wagner and colleagues found that preoperative signal coupling between the ipsilateral hippocampus and superior temporal gyrus during a resting state scan was negatively correlated with disease duration and positively correlated with postoperative decline in memory [113]. Of interest, the primary resting state network, known as the default mode network $(\mathrm{DMN})$, is largely coextensive with the networks involved in autobiographical recall and internal mentation [114] and researchers have begun to examine how connectivity in DMN components may relate to clinical parameters (e.g., seizure duration, cognitive deficits) in TLE [115-117].

\section{Conceptual and Methodological Caveats in fMRI}

There are a number of important limitations of functional activation techniques that require consideration. First, activations reflect correlations between some characteristic of the underlying neural substrate and behavior but this does not mean that an activated region is essential in performing the relevant task. Thus, convergence with other methods (stimulation, inactivation, and lesion) is crucial in making such inferences. Second, the careful selection of tasks is imperative, particularly as studies typically use a "subtraction" method that attempts to eliminate influences from all but the critical process, yet identification of the appropriate "control" conditions may be quite complicated [118]. Also, the magnitude of these correlations can be artificially inflated in reports, when additional inferential statistics (such as correlations of activation magnitude with behavior) are performed on regions identified in a firstpass analysis, the so-called "double-dipping" phenomenon [119]. As noted above, there may be a very complex relationship between magnitude of activation (or another BOLD parameter of interest) and clinical status and there may also be a function of a host of factors (estimates of structural integrity, etiology of and duration of disease state, and cerebral reserve) that we are only beginning to address in our analyses. The influence of differential task performance is also a crucial variable in that the impact of inadequate task performance on differential activation is exceptionally difficult to assess [120]. The use of task-independent patterns of connectivity, such as those characterized by the DMN, may mitigate some of these methodological problems, but this is unlikely to permit exploration of the full range of cognitive capacities of interest in presurgical planning. Finally, we do not know the extent to which seizures, mesial temporal sclerosis, or antiepileptic drugs may compromise (either globally or locally) the neurovascular coupling that is crucial for observing a BOLD effect [121]. Despite these caveats, fMRI holds considerable promise, with no significant risk to patients, to substantially enhance our knowledge of how language and memory networks change in TLE. Furthermore, there is considerable clinical promise in that one can derive the positive predictive value of the mapping techniques in regard to risk to memory given that results are not used in surgical decision making, unlike the IAP for which test results constrain the treatment offered.

\section{Conclusion}

Neuropsychological assessment remains a critical part of surgical planning for TLE, and it is evolving in precision of diagnosis/prediction by incorporating novel concepts and techniques from cognitive neuroscience and functional imaging. This evolution will be shaped by our appreciation of the fact that even a focal seizure disorder can have widespread effects on anatomy and function and acknowledgement that TLE may represent a paradigm case of subtle functional reorganization that requires us to be informed about the integrity and degeneracy [122] of networks supporting complex cognitive operations.

\section{References}

[1] D. W. Loring, "History of neuropsychology through epilepsy eyes," Archives of Clinical Neuropsychology, vol. 25, no. 4, pp. 259-273, 2010.

[2] B. Bell, J. J. Lin, M. Seidenberg, and B. Hermann, "The neurobiology of cognitive disorders in temporal lobe epilepsy," Nature Reviews Neurology, vol. 7, no. 3, pp. 154-164, 2011. 
[3] M. Jones-Gotman, M. L. Smith, G. L. Risse et al., "The contribution of neuropsychology to diagnostic assessment in epilepsy," Epilepsy and Behavior, vol. 18, no. 1-2, pp. 3-12, 2010.

[4] W. PENFIELD and B. MILNER, "Memory deficit produced by bilateral lesions in the hippocampal zone," Archives of Neurology and Psychiatry, vol. 79, no. 5, pp. 475-497, 1958.

[5] W. B. Scoville and B. Milner, "Loss of recent memory after bilateral hippocampal lesions," Journal of Neurology, Neurosurgery, and Psychiatry, vol. 20, no. 1, pp. 11-21, 1957.

[6] E. Strauss, E. M. S. Sherman, and O. Spreen, A Compendium of Neuropsychological Tests : Administration, Norms, and Commentary, Oxford University Press, Oxford, UK, 3rd edition, 2006.

[7] B. Milner, "Memory and the medial temporal regions of the brain," in Biology of Memory, K. H. Pribram and D. E. Broadbent, Eds., pp. 29-50, Academic Press, NewYork, NY, USA, 1970.

[8] C. Helmstaedter, T. Grunwald, K. Lehnertz, U. Gleißner, and C. E. Elger, "Differential involvement of left temporolateral and temporomesial structures in verbal declarative learning and memory: evidence from temporal lobe epilepsy," Brain and Cognition, vol. 35, no. 1, pp. 110-131, 1997.

[9] K. J. Sass, C. P. Buchanan, S. Kraemer, M. Westerveld, J. H. Kim, and D. D. Spencer, "Verbal memory impairment resulting from hippocampal neuron loss among epileptic patients with structural lesions," Neurology, vol. 45, no. 12, pp. 2154-2158, 1995.

[10] M. M. Saling, "Verbal memory in mesial temporal lobe epilepsy: beyond material specificity," Brain, vol. 132, no. 3, pp. 570-582, 2009.

[11] R. Rausch and T. L. Babb, "Hippocampal neuron loss and memory scores before and after temporal lobe surgery for epilepsy," Archives of Neurology, vol. 50, no. 8, pp. 812-817, 1993.

[12] U. Gleißner, C. Helmstaedter, and C. E. Elger, "Right hippocampal contribution to visual memory: a presurgical and postsurgical study in patients with temporal lobe epilepsy," Journal of Neurology Neurosurgery and Psychiatry, vol. 65, no. 5, pp. 665-669, 1998.

[13] C. Helmstaedter, C. Pohl, A. Hufnagel, and C. E. Elger, "Visual learning deficits in nonresected patients with right temporal lobe epilepsy," Cortex, vol. 27, no. 4, pp. 547-555, 1991.

[14] S. A. Baxendale, P. J. Thompson, and W. Van Paesschen, "A test of spatial memory and its clinical utility in the presurgical investigation of temporal lobe epilepsy patients," Neuropsychologia, vol. 36, no. 7, pp. 591-602, 1998.

[15] Y. Glikmann-Johnston, M. M. Saling, J. Chen, K. A. Cooper, R. J. Beare, and D. C. Reutens, "Structural and functional correlates of unilateral mesial temporal lobe spatial memory impairment," Brain, vol. 131, no. 11, pp. 3006-3018, 2008.

[16] E. Tulving, "Episodic and semantic memory," in Organization of Memory, E. Tulving and W. Donaldson, Eds., pp. 381-403, Academic Press, New York, NY, USA, 1972.

[17] K. S. Graham and J. R. Hodges, "Differentiating the roles of the hippocampal complex and the neocortex in longterm memory storage: evidence from the study of semantic dementia and Alzheimer's disease," Neuropsychology, vol. 11, no. 1, pp. 77-89, 1997.

[18] D. L. Greenberg and M. Verfaellie, "Interdependence of episodic and semantic memory: evidence from neuropsychology," Journal of the International Neuropsychological Society, vol. 16, no. 5, pp. 748-753, 2010.
[19] D. W. Loring, E. Strauss, B. P. Hermann et al., "Differential neuropsychological test sensitivity to left temporal lobe epilepsy," Journal of the International Neuropsychological Society, vol. 14, no. 3, pp. 394-400, 2008.

[20] M. M. Saling, S. F. Berkovic, M. F. O’Shea, R. M. Kalnins, D. G. Darby, and P. F. Bladin, "Lateralization of verbal memory and unilateral hippocampal sclerosis: evidence of taskspecific effects," Journal of Clinical and Experimental Neuropsychology, vol. 15, no. 4, pp. 608-618, 1993.

[21] D. L. Greenberg, M. M. Keane, L. Ryan, and M. Verfaellie, "Impaired category fluency in medial temporal lobe amnesia: the role of episodic memory," Journal of Neuroscience, vol. 29, no. 35, pp. 10900-10908, 2009.

[22] L. Ryan, C. Cox, S. M. Hayes, and L. Nadel, "Hippocampal activation during episodic and semantic memory retrieval: comparing category production and category cued recall," Neuropsychologia, vol. 46, no. 8, pp. 2109-2121, 2008.

[23] U. Gleissner and C. E. Elger, "The hippocampal contribution to verbal fluency in patients with temporal lobe epilepsy," Cortex, vol. 37, no. 1, pp. 55-63, 2001.

[24] R. Westmacott, S. E. Black, M. Freedman, and M. Moscovitch, "The contribution of autobiographical significance to semantic memory: evidence from Alzheimer's disease, semantic dementia, and amnesia," Neuropsychologia, vol. 42, no. 1, pp. 25-48, 2004.

[25] I. V. Viskontas, M. P. McAndrews, and M. Moscovitch, "Memory for famous people in patients with unilateral temporal lobe epilepsy and excisions," Neuropsychology, vol. 16, no. 4, pp. 472-480, 2002.

[26] M. D. Rugg and A. P. Yonelinas, "Human recognition memory: a cognitive neuroscience perspective," Trends in Cognitive Sciences, vol. 7, no. 7, pp. 313-319, 2003.

[27] A. P. Yonelinas, "The nature of recollection and familiarity: a review of 30 years of research," Journal of Memory and Language, vol. 46, no. 3, pp. 441-517, 2002.

[28] M. Cohn, M. P. McAndrews, and M. Moscovitch, "Associative reinstatement: a novel approach to assessing associative memory in patients with unilateral temporal lobe excisions," Neuropsychologia, vol. 47, no. 13, pp. 2989-2994, 2009.

[29] M. St-Laurent, M. Moscovitch, B. Levine, and M. P. McAndrews, "Determinants of autobiographical memory in patients with unilateral temporal lobe epilepsy or excisions," Neuropsychologia, vol. 47, no. 11, pp. 2211-2221, 2009.

[30] L. Thaiss and M. Petrides, "Source versus content memory in patients with a unilateral frontal cortex or a temporal lobe excision," Brain, vol. 126, no. 5, pp. 1112-1126, 2003.

[31] I. V. Viskontas, M. P. McAndrews, and M. Moscovitch, "Remote episodic memory deficits in patients with unilateral temporal lobe epilepsy and excisions," Journal of Neuroscience, vol. 20, no. 15, pp. 5853-5857, 2000.

[32] M. Moran, M. Seidenberg, D. Sabsevitz, S. Swanson, and B. Hermann, "The acquisition of face and person identity information following anterior temporal lobectomy," Journal of the International Neuropsychological Society, vol. 11, no. 3, pp. 237-248, 2005.

[33] M. L. Smith, M. Bigel, and L. A. Miller, "Visual pairedassociate learning: in search of material-specific effects in adult patients who have undergone temporal lobectomy," Epilepsy and Behavior, vol. 20, no. 2, pp. 326-330, 2011.

[34] D. A. Moscovitch and M. P. McAndrews, "Material-specific deficits in 'remembering' in patients with unilateral temporal lobe epilepsy and excisions," Neuropsychologia, vol. 40, no. 8, pp. 1335-1342, 2002. 
[35] B. Bowles, C. Crupi, S. M. Mirsattari et al., "Impaired familiarity with preserved recollection after anterior temporallobe resection that spares the hippocampus," Proceedings of the National Academy of Sciences of the United States of America, vol. 104, no. 41, pp. 16382-16387, 2007.

[36] J. L. McClelland, B. L. McNaughton, and R. C. O'Reilly, “Why there are complementary learning systems in the hippocampus and neocortex: insights from the successes and failures of connectionist models of learning and memory," Psychological Review, vol. 102, no. 3, pp. 419-457, 1995.

[37] R. C. O’Reilly and J. W. Rudy, “Conjunctive representations in learning and memory: principles of cortical and hippocampal function," Psychological Review, vol. 108, no. 2, pp. 311-345, 2001.

[38] K. A. Norman and R. C. O’Reilly, "Modeling hippocampal and neocortical contributions to recognition memory: a complementary-learning-systems approach," Psychological Review, vol. 110, no. 4, pp. 611-646, 2003.

[39] J. S. Holdstock, A. R. Mayes, N. Roberts et al., "Under what conditions is recognition spared relative to recall after selective hippocampal damage in humans?" Hippocampus, vol. 12, no. 3, pp. 341-351, 2002.

[40] C. B. Kirwan and C. E. L. Stark, "Overcoming interference: an fMRI investigation of pattern separation in the medial temporal lobe," Learning and Memory, vol. 14, no. 9, pp. 625633, 2007.

[41] R. G. Morris, S. Abrahams, A. D. Baddeley, and C. E. Polkey, "Doors and people: visual and verbal memory after unilateral temporal lobectomy," Neuropsychology, vol. 9, no. 4, pp. 464469, 1995.

[42] T. M. C. Lee, J. T. H. Yip, and M. Jones-Gotman, "Memory deficits after resection from left or right anterior temporal lobe in humans: a meta-analytic review," Epilepsia, vol. 43, no. 3, pp. 283-291, 2002.

[43] Y. Dudai, "The neurobiology of consolidations, or, how stable is the engram?" Annual Review of Psychology, vol. 55, pp. 5186, 2004.

[44] M. Jones-Gotman, R. J. Zatorre, A. Olivier et al., "Learning and retention of words and designs following excision from medial or lateral temporal lobe structures," Neuropsychologia, vol. 35, no. 7, pp. 963-973, 1997.

[45] N. Muhlert, R. A. Grünewald, N. M. Hunkin et al., "Accelerated long-term forgetting in temporal lobe but not idiopathic generalised epilepsy," Neuropsychologia, vol. 49, no. 9, pp. 2417-2426, 2011.

[46] H. Wilkinson, J. S. Holdstock, G. Baker, A. Herbert, F. Clague, and J. J. Downes, "Long-term accelerated forgetting of verbal and non-verbal information in temporal lobe epilepsy," Cortex, 2011.

[47] S. Baxendale, P. Thompson, W. Harkness, and J. Duncan, "Predicting memory decline following epilepsy surgery: a multivariate approach," Epilepsia, vol. 47, no. 11, pp. 18871894, 2006.

[48] B. Milner, C. Branch, and T. Rasmussen, "Study of shortterm memory after intracarotid injection of sodium amytal," Transactions of the American Neurological Association, vol. 87, pp. 224-226, 1962.

[49] J. A. Wada, "Clinical experimental observations of carotid artery injections of sodium amytal," Brain and Cognition, vol. 33, no. 1, pp. 11-13, 1997.

[50] C. S. Kubu, J. P. Girvin, R. S. McLachlan, M. Pavol, and M. C. S. Harnadek, "Does the intracarotid amobarbital procedure predict global amnesia after temporal lobectomy?" Epilepsia, vol. 41, no. 10, pp. 1321-1329, 2000.
[51] J. Mani, R. Busch, C. Kubu, P. Kotagal, U. Shah, and D. Dinner, "Wada memory asymmetry scores and postoperative memory outcome in left temporal epilepsy," Seizure, vol. 17, no. 8, pp. 691-698, 2008.

[52] T. T. Lineweaver, H. H. Morris, R. I. Naugle, I. M. Najm, B. Diehl, and W. Bingaman, "Evaluating the contributions of state-of-the-art assessment techniques to predicting memory outcome after unilateral anterior temporal lobectomy," Epilepsia, vol. 47, no. 11, pp. 1895-1903, 2006.

[53] N. Elshorst, B. Pohlmann-Eden, S. Horstmann, R. Schulz, F. Woermann, and M. P. McAndrews, "Postoperative memory prediction in left temporal lobe epilepsy: the Wada test is of no added value to preoperative neuropsychological assessment and MRI," Epilepsy and Behavior, vol. 16, no. 2, pp. 335-340, 2009.

[54] S. Baxendale, "The Wada test," Current Opinion in Neurology, vol. 22, no. 2, pp. 185-189, 2009.

[55] S. Ogawa, D. W. Tank, R. Menon et al., "Intrinsic signal changes accompanying sensory stimulation: functional brain mapping with magnetic resonance imaging," Proceedings of the National Academy of Sciences of the United States of America, vol. 89, no. 13, pp. 5951-5955, 1992.

[56] K. K. Kwong, J. W. Belliveau, D. A. Chesler et al., "Dynamic magnetic resonance imaging of human brain activity during primary sensory stimulation," Proceedings of the National Academy of Sciences of the United States of America, vol. 89, no. 12 , pp. 5675-5679, 1992.

[57] J. Duncan, "The current status of neuroimaging for epilepsy," Current Opinion in Neurology, vol. 22, no. 2, pp. 179-184, 2009.

[58] M. Richardson, "Current themes in neuroimaging of epilepsy: brain networks, dynamic phenomena, and clinical relevance," Clinical Neurophysiology, vol. 121, no. 8, pp. 11531175,2010

[59] T. Rasmussen and B. Milner, "The role of early leftbrain injury in determining lateralization of cerebral speech functions," Annals of the New York Academy of Sciences, vol. 299, pp. 355-369, 1977.

[60] R. P. Woods, C. B. Dodrill, and G. A. Ojemann, "Brain injury, handedness, and speech lateralization in a series of amobarbital studies," Annals of Neurology, vol. 23, no. 5, pp. 510-518, 1988.

[61] F. Liégeois, A. Connelly, C. H. Salmond, D. G. Gadian, F. Vargha-Khadem, and T. Baldeweg, "A direct test for lateralization of language activation using fMRI: comparison with invasive assessments in children with epilepsy," NeuroImage, vol. 17, no. 4, pp. 1861-1867, 2002.

[62] D. S. Kadis, E. N. Kerr, J. T. Rutka, O. C. Snead III, S. K. Weiss, and M. L. Smith, "Pathology type does not predict language lateralization in children with medically intractable epilepsy," Epilepsia, vol. 50, no. 6, pp. 1498-1504, 2009.

[63] M. V. Baciu, J. M. Watson, L. Maccotta et al., "Evaluating functional MRI procedures for assessing hemispheric language dominance in neurosurgical patients," Neuroradiology, vol. 47, no. 11, pp. 835-844, 2005.

[64] J. P. Szaflarski, S. K. Holland, L. M. Jacola, C. Lindsell, M. D. Privitera, and M. Szaflarski, "Comprehensive presurgical functional MRI language evaluation in adult patients with epilepsy," Epilepsy and Behavior, vol. 12, no. 1, pp. 74-83, 2008.

[65] J. Arora, K. Pugh, M. Westerveld, S. Spencer, D. D. Spencer, and R. Todd Constable, "Language lateralization in epilepsy patients: FMRI validated with the Wada procedure," Epilepsia, vol. 50, no. 10, pp. 2225-2241, 2009. 
[66] W. D. Gaillard, L. Balsamo, B. Xu et al., "fMRI language task panel improves determination of language dominance," Neurology, vol. 63, no. 8, pp. 1403-1408, 2004.

[67] J. E. Desmond, J. M. Sum, A. D. Wagner et al., "Functional MRI measurement of language lateralization in Wada-tested patients," Brain, vol. 118, no. 6, pp. 1411-1419, 1995.

[68] S. Lehéricy, L. Cohen, B. Bazin et al., "Functional MR evaluation of temporal and frontal language dominance compared with the Wada test," Neurology, vol. 54, no. 8, pp. 1625-1633, 2000.

[69] L. Hertz-Pannier, W. D. Gaillard, S. H. Mott et al., "Noninvasive assessment of language dominance in children and adolescents with functional MRI: a preliminary study," Neurology, vol. 48, no. 4, pp. 1003-1012, 1997.

[70] F. G. Woermann, H. Jokeit, R. Luerding et al., "Language lateralization by Wada test and fMRI in 100 patients with epilepsy," Neurology, vol. 61, no. 5, pp. 699-701, 2003.

[71] J. A. Springer, J. R. Binder, T. A. Hammeke et al., "Language dominance in neurologically normal and epilepsy subjects. A functional MRI study," Brain, vol. 122, no. 11, pp. 2033-2045, 1999.

[72] J. E. Adcock, R. G. Wise, J. M. Oxbury, S. M. Oxbury, and P. M. Matthews, "Quantitative fMRI assessment of the differences in lateralization of language-related brain activation in patients with temporal lobe epilepsy," NeuroImage, vol. 18, no. 2, pp. 423-438, 2003.

[73] N. L. Voets, J. E. Adcock, R. Stacey et al., "Functional and structural changes in the memory network associated with left temporal lobe epilepsy," Human Brain Mapping, vol. 30, no. 12, pp. 4070-4081, 2009.

[74] T. Benke, B. Köylü, P. Visani et al., "Language lateralization in temporal lobe epilepsy: a comparison between fMRI and the Wada test," Epilepsia, vol. 47, no. 8, pp. 1308-1319, 2006.

[75] L. Thivard, J. Hombrouck, S. Tézenas Du Montcel et al., "Productive and perceptive language reorganization in temporal lobe epilepsy," NeuroImage, vol. 24, no. 3, pp. 841-851, 2005.

[76] C. H. Kim, C. K. Chung, B.-B. Koo, J.-M. Lee, J. S. Kim, and S. K. Lee, "Changes in language pathways in patients with temporal lobe epilepsy: diffusion tensor imaging analysis of the uncinate and arcuate fasciculi," World Neurosurgery, vol. 75, no. 3-4, pp. 509-516, 2011.

[77] G. J. M. Rutten, N. F. Ramsey, P.C. van Rijen, W. C. Alpherts, and C. W. M. van Veelen, "fMRI-determined language lateralization in patients with unilateral or mixed language dominance according to the Wada Test," NeuroImage, vol. 17, pp. 447-460, 2002.

[78] A. Sharan, Y. C. Ooi, J. Langfitt, and M. R. Sperling, "Intracarotid amobarbital procedure for epilepsy surgery," Epilepsy and Behavior, vol. 20, no. 2, pp. 209-213, 2011.

[79] D. S. Sabsevitz, S. J. Swanson, T. A. Hammeke et al., "Use of preoperative functional neuroimaging to predict language deficits from epilepsy surgery," Neurology, vol. 60, no. 11, pp. 1788-1792, 2003.

[80] M. V. Baciu, J. M. Watson, K. B. McDermott et al., "Functional MRI reveals an interhemispheric dissociation of frontal and temporal language regions in a patient with focal epilepsy," Epilepsy and Behavior, vol. 4, no. 6, pp. 776-780, 2003.

[81] M. L. Ries, F. A. Boop, M. L. Griebel et al., "Functional MRI and Wada determination of language lateralization: a case of crossed dominance," Epilepsia, vol. 45, no. 1, pp. 85-89, 2004.

[82] R. L. Billingsley, M. P. McAndrews, A. P. Crawley, and D. J. Mikulis, "Functional MRI of phonological and semantic processing in temporal lobe epilepsy," Brain, vol. 124, no. 6, pp. 1218-1227, 2001.

[83] E. Cousin, M. Baciu, C. Pichat, P. Kahane, and J. F. Le Bas, "Functional MRI evidence for language plasticity in adult epileptic patients: preliminary results," Neuropsychiatric Disease and Treatment, vol. 4, no. 1, pp. 235-246, 2008.

[84] A. B. Waites, R. S. Briellmann, M. M. Saling, D. F. Abbott, and G. D. Jackson, "Functional connectivity networks are disrupted in left temporal lobe epilepsy," Annals of Neurology, vol. 59, no. 2, pp. 335-343, 2006.

[85] A. B. Protzner and M. P. McAndrews, "Network alterations supporting word retrieval in patients with medial temporal lobe epilepsy," Journal of Cognitive Neuroscience, vol. 23, no. 9, pp. 2606-2619, 2011.

[86] S. Kennepohl, V. Sziklas, K. E. Garver, D. D. Wagner, and M. Jones-Gotman, "Memory and the medial temporal lobe: hemispheric specialization reconsidered," NeuroImage, vol. 36, no. 3, pp. 969-978, 2007.

[87] J. A. Detre, L. Maccotta, D. King et al., "Functional MRI lateralization of memory in temporal lobe epilepsy," Neurology, vol. 50, no. 4, pp. 926-932, 1998.

[88] H. Jokeit, M. Okujava, and F. G. Woermann, "Memory fMRI lateralizes temporal lobe epilepsy," Neurology, vol. 57, no. 10, pp. 1786-1793, 2001.

[89] D. R. Addis, M. Moscovitch, and M. P. McAndrews, "Consequences of hippocampal damage across the autobiographical memory network in left temporal lobe epilepsy," Brain, vol. 130, no. 9, pp. 2327-2342, 2007.

[90] M. C. Cheung, A. S. Chan, J. M. K. Lam, and Y. L. Chan, "Preand postoperative fMRI and clinical memory performance in temporal lobe epilepsy," Journal of Neurology, Neurosurgery and Psychiatry, vol. 80, no. 10, pp. 1099-1106, 2009.

[91] R. Beisteiner, K. Drabeck, T. Foki et al., "Does clinical memory fMRI provide a comprehensive map of medial temporal lobe structures?" Experimental Neurology, vol. 213, no. 1, pp. 154-162, 2008.

[92] S. Dupont, P. F. Van De Moortele, S. Samson et al., "Episodic memory in left temporal lobe epilepsy: a functional MRI study," Brain, vol. 123, no. 8, pp. 1722-1732, 2000.

[93] A. J. Golby, R. A. Poldrack, J. Illes, D. Chen, J. E. Desmond, and J. D. E. Gabrieli, "Memory lateralization in medial temporal lobe epilepsy assessed by functional MRI," Epilepsia, vol. 43, no. 8, pp. 855-863, 2002.

[94] P. S. F. Bellgowan, J. R. Binder, S. J. Swanson et al., "Side of seizure focus predicts left medial temporal lobe activation during verbal encoding," Neurology, vol. 51, no. 2, pp. 479484, 1998.

[95] R. Beisteiner, N. Klinger, I. Höllinger et al., "How much are clinical fMRI reports influenced by standard postprocessing methods? An investigation of normalization and region of interest effects in the medial temporal lobe," Human Brain Mapping, vol. 31, no. 12, pp. 1951-1966, 2010.

[96] S. B. Bonelli, R. H. W. Powell, M. Yogarajah et al., "Imaging memory in temporal lobe epilepsy: predicting the effects of temporal lobe resection," Brain, vol. 133, no. 4, pp. 11861199, 2010.

[97] M. L. Rabin, V. M. Narayan, D. Y. Kimberg et al., "Functional MRI predicts post-surgical memory following temporal lobectomy," Brain, vol. 127, no. 10, pp. 2286-2298, 2004.

[98] M. P. Richardson, B. A. Strange, P. J. Thompson, S. A. Baxendale, J. S. Duncan, and R. J. Dolan, "Pre-operative verbal memory fMRI predicts post-operative memory decline after left temporal lobe resection," Brain, vol. 127, no. 11, pp. 2419-2426, 2004. 
[99] M. P. Richardson, B. A. Strange, J. S. Duncan, and R. J. Dolan, "Memory fMRI in left hippocampal sclerosis: optimizing the approach to predicting postsurgical memory," Neurology, vol. 66, no. 5, pp. 699-705, 2006.

[100] L. Frings, K. Wagner, U. Halsband, R. Schwarzwald, J. Zentner, and A. Schulze-Bonhage, "Lateralization of hippocampal activation differs between left and right temporal lobe epilepsy patients and correlates with postsurgical verbal learning decrement," Epilepsy Research, vol. 78, no. 2-3, pp. 161-170, 2008.

[101] H. W. R. Powell, M. P. Richardson, M. R. Symms et al., "Preoperative fMRI predicts memory decline following anterior temporal lobe resection," Journal of Neurology, Neurosurgery and Psychiatry, vol. 79, no. 6, pp. 686-693, 2008.

[102] J. R. Binder, S. J. Swanson, D. S. Sabsevitz, T. A. Hammeke, M. Raghavan, and W. M. Mueller, "A comparison of two fMRI methods for predicting verbal memory decline after left temporal lobectomy: language lateralization versus hippocampal activation asymmetry," Epilepsia, vol. 51, no. 4, pp. 618-626, 2010.

[103] J. R. Binder, "Preoperative prediction of verbal episodic memory outcome using fMRI," Neurosurgery Clinics of North America, vol. 22, no. 2, pp. 219-232, 2011.

[104] S. Dupont, E. Duron, S. Samson et al., "Functional MR imaging or wada test: which is the better predictor of individual postoperative memory outcome?" Radiology, vol. 255, no. 1, pp. 128-134, 2010.

[105] E. Svoboda, M. C. McKinnon, and B. Levine, "The functional neuroanatomy of autobiographical memory: a metaanalysis," Neuropsychologia, vol. 44, no. 12, pp. 2189-2208, 2006.

[106] E. A. Maguire, "Neuroimaging studies of autobiographical event memory," Philosophical Transactions of the Royal Society $B$, vol. 356, no. 1413, pp. 1441-1451, 2001.

[107] E. A. Maguire, F. Vargha-Khadem, and M. Mishkin, "The effects of bilateral hippocampal damage on fMRI regional activations and interactions during memory retrieval," Brain, vol. 124, no. 6, pp. 1156-1170, 2001.

[108] E. A. Maguire, C. D. Frith, P. Rudge, and L. Cipolotti, "The effect of adult-acquired hippocampal damage on memory retrieval: an fMRI study," NeuroImage, vol. 27, no. 1, pp. 146152, 2005.

[109] E. Guedj, G. Bettus, E. J. Barbeau et al., "Hyperactivation of parahippocampal region and fusiform gyrus associated with successful encoding in medial temporal lobe epilepsy," Epilepsia, vol. 52, no. 6, pp. 1100-1109, 2011.

[110] B. C. Dickerson, D. H. Salat, D. N. Greve et al., "Increased hippocampal activation in mild cognitive impairment compared to normal aging and AD," Neurology, vol. 65, no. 3, pp. 404-411, 2005.

[111] R. A. Sperling, B. C. Dickerson, M. Pihlajamaki et al., "Functional alterations in memory networks in early alzheimer's disease," NeuroMolecular Medicine, vol. 12, no. 1, pp. 27-43, 2010.

[112] J. R. Binder, D. S. Sabsevitz, S. J. Swanson, T. A. Hammeke, M. Raghavan, and W. M. Mueller, "Use of preoperative functional MRI to predict verbal memory decline after temporal lobe epilepsy surgery," Epilepsia, vol. 49, no. 8, pp. 1377-1394, 2008.

[113] K. Wagner, L. Frings, U. Halsband et al., "Hippocampal functional connectivity reflects verbal episodic memory network integrity," NeuroReport, vol. 18, no. 16, pp. 1719-1723, 2007.

[114] R. L. Buckner, J. R. Andrews-Hanna, and D. L. Schacter, "The brain's default network: anatomy, function, and relevance to disease," Annals of the New York Academy of Sciences, vol. 1124, pp. 1-38, 2008.

[115] L. Frings, A. Schulze-Bonhage, J. Spreer, and K. Wagner, "Remote effects of hippocampal damage on default network connectivity in the human brain," Journal of Neurology, vol. 256, no. 12, pp. 2021-2029, 2009.

[116] W. Liao, Z. Zhang, Z. Pan et al., "Altered functional connectivity and small-world in mesial temporal lobe epilepsy," PLoS One, vol. 5, no. 1, Article ID e8525, 2010.

[117] Z. Zhang, G. Lu, Y. Zhong et al., "Altered spontaneous neuronal activity of the default-mode network in mesial temporal lobe epilepsy," Brain Research, vol. 1323, pp. 152-160, 2010.

[118] J. R. Binder, "Functional MRI is a valid noninvasive alternative to Wada testing," Epilepsy and Behavior, vol. 20, no. 2, pp. 214-222, 2011.

[119] N. Kriegeskorte, W. K. Simmons, P. S. Bellgowan, and C. I. Baker, "Circular analysis in systems neuroscience: the dangers of double dipping," Nature Neuroscience, vol. 12, no. 5, pp. 535-540, 2009.

[120] C. J. Price and K. J. Friston, "Functional imaging studies of neuropsychological patients: applications and limitations," Neurocase, vol. 8, no. 5, pp. 345-354, 2002.

[121] G. Bettus, J. -P. Ranjeva, F. Wendling et al., "Interictal functional connectivity of human epileptic networks assessed by intracerebral EEG and BOLD signal fluctuations," PLoS One, vol. 6, no. 5, Article ID e20071, 2011.

[122] C. J. Price and K. J. Friston, "Degeneracy and cognitive anatomy," Trends in Cognitive Sciences, vol. 6, no. 10, pp. 416-421, 2002. 


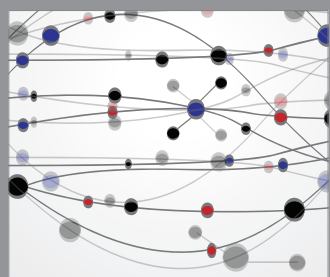

The Scientific World Journal
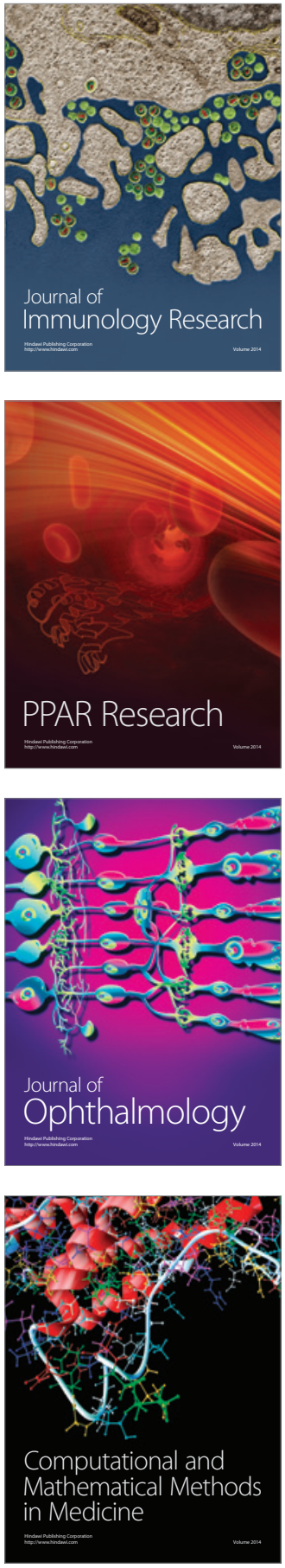

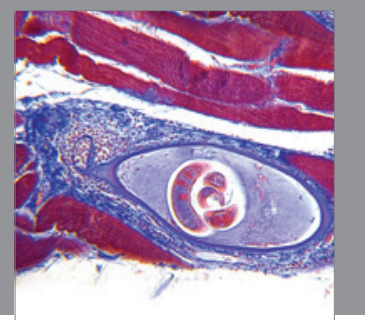

Gastroenterology

Research and Practice
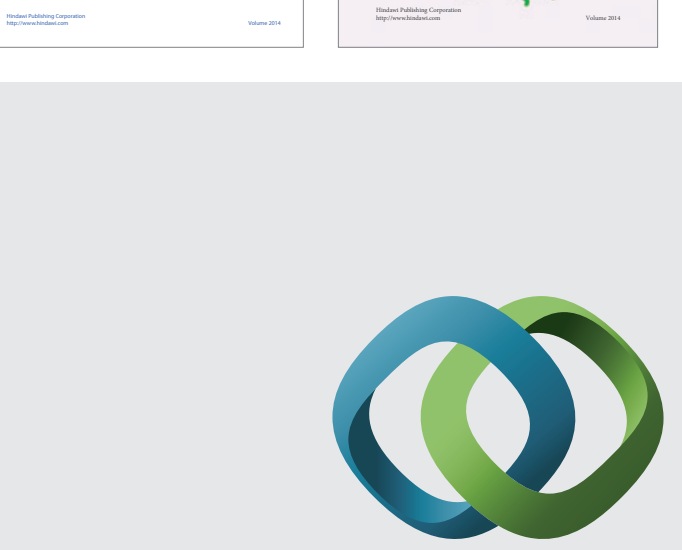

\section{Hindawi}

Submit your manuscripts at

http://www.hindawi.com
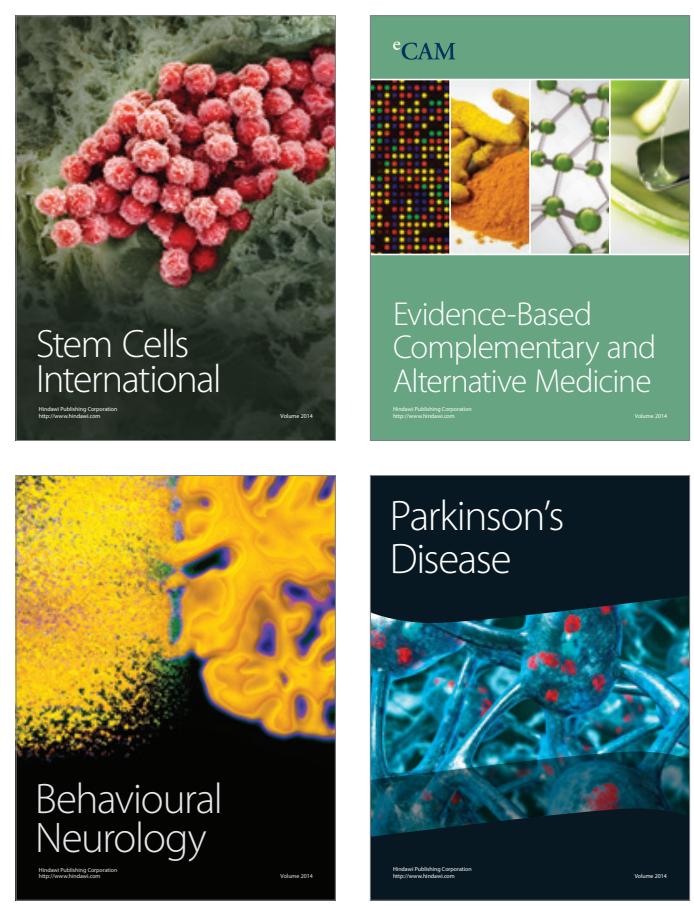

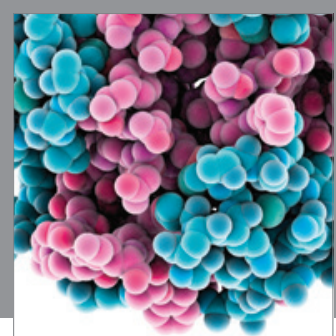

Journal of
Diabetes Research

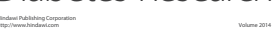

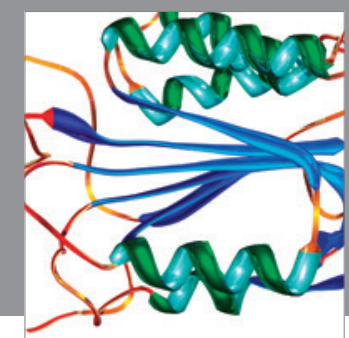

Disease Markers
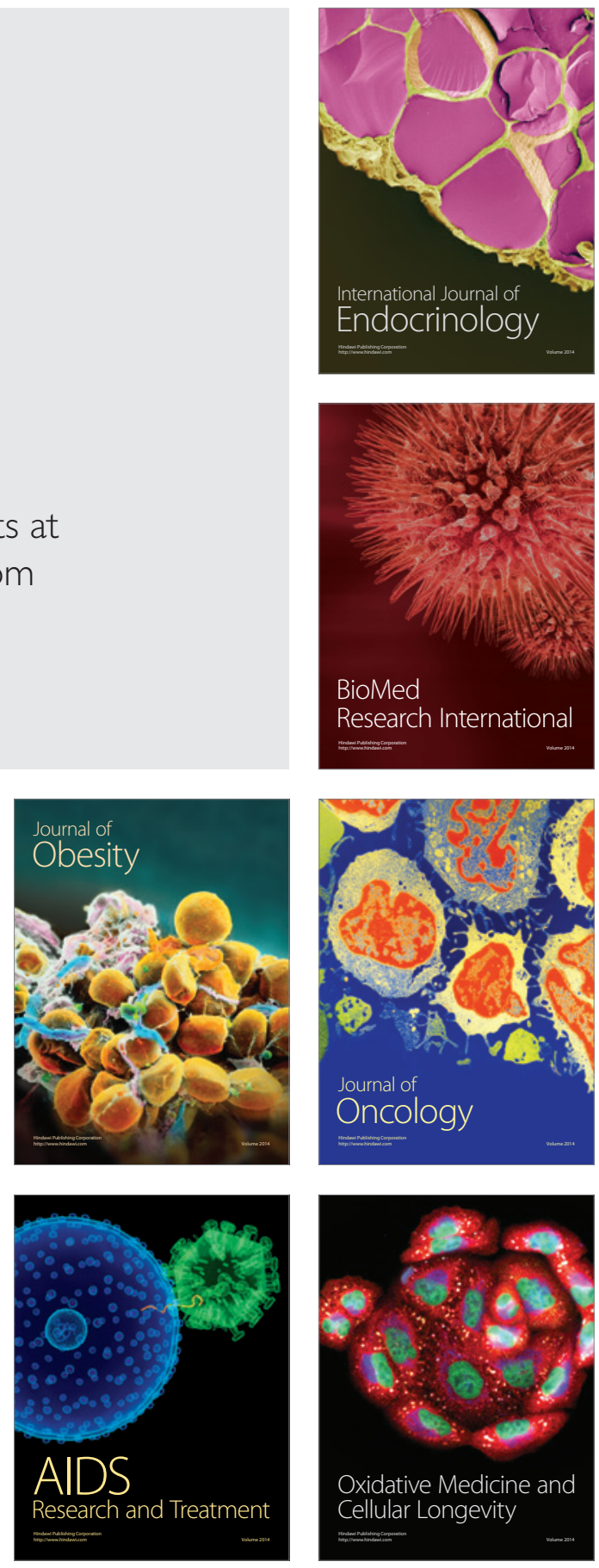